\title{
Preface to the requirements engineering special issue on selected papers from RE'19
}

\author{
Kelly Blincoe ${ }^{1} \cdot$ Daniela Damian $^{2} \cdot$ Anna Perini $^{3}$
}

Published online: 19 October 2020

๑) Springer-Verlag London Ltd., part of Springer Nature 2020

Welcome to this special issue that includes papers on the latest advances in Requirements Engineering (RE).

The selected studies extend research presented at RE'19, the 27th IEEE International Requirements Engineering Conference. The RE conference is the premier international forum for researchers, practitioners, educators, and students to present and discuss the most recent innovations, experiences, and concerns in the discipline of requirements engineering. RE'19 had the theme "RE and Collective Intelligence in the Days of AI" and was held in Jeju Island, South Korea, from September 23 to 27, 2019 [1].

Six papers describing new RE techniques and guidelines stood out at RE' 19 as exceptional contributions. The authors of these candidate papers were invited to submit extended manuscripts for this special issue. The submitted manuscripts were each rigorously peer reviewed, and four were accepted for inclusion in this special issue.

Summary of the papers The first article, "SaPeer and ReverseSaPeer: Teaching Requirements Elicitation Interviews with Role-playing and Role Reversal" by Alessio Ferrari, Paola Spoletini, Muneera Bano, and Didar Zowghi, presents two new pedagogical approaches, named SaPeer and ReverseSaPeer, to teach interview skills for requirements elicitation. The two approaches use role-playing and role reversal to provide first-hand experience in requirements elicitation from the perspective of both the interviewer and the interviewee. The two methods were found to be useful by students. The authors share their pedagogical material,

Kelly Blincoe

k.blincoe@auckland.ac.nz

1 Department of Electrical, Computer, and Software Engineering, University of Auckland, 5 Grafton Road, Auckland 1010, New Zealand

2 Department of Computer Science, University of Victoria, 3800 Finnerty Road, Victoria, BC V8P 5C2, Canada

3 Fondazione Bruno Kessler, Via Sommarive, 18 - POVO, 38123 Trento, Italy enabling other educators to apply these approaches in their own teaching.

The second article, "An Efficient Approach for Reviewing Security-Related Aspects in Agile Requirements Specifications of Web Applications" by Hugo Villamizar, Marcos Kalinowski, Alessandro Garcia, and Daniel Mendez, introduces an approach to support software engineers in verifying security requirements in agile requirement specifications of web applications during requirement inspections. The approach, which generates a reading technique to support requirement inspectors in detecting security requirement defects, was found to improve the effectiveness and efficiency of novice software engineers. The approach can help ensure better defined security-related software requirements, enabling more secure software products.

The third article, "Requirements Engineering for Sustainability: An Awareness Framework for Designing Software Systems for a Better Tomorrow" by Leticia Duboc, Birgit Penzenstadler, Jari Porras, Sedef Akinli Kocak, Stefanie Betz, Ruzanna Chitchyan, Ola Leifler, Norbert Seyff, and Colin C. Venters, proposes a question-based framework, called the Sustainability Awareness Framework, for raising awareness of software practitioners on the effects of software systems on sustainability. As new software systems are developed, they can impact our society, economy and environment, and software requirement engineers should consider the implications on sustainability when designing software requirements. The proposed framework was shown to help facilitate discussions on how software systems can impact sustainability. Thus, it can help software teams design software systems with sustainability in mind.

The fourth article, "Explainability as a Non-Functional Requirement: Challenges and Recommendations" by Larissa Chazette and Kurt Schneider, presents results of a survey with 107 software users on their perceptions of transparency of software systems and explanations embedded within software systems. The results show that built-in explanations can help software systems achieve transparency and 
build trust. However, these built-in explanations can also have negative effects on non-functional requirements like usability. The paper provides concrete recommendations to ensure the explanations are well designed. Following these guidelines can help promote more transparent software without sacrificing usability.

Collectively, these four contributions represent diverse and relevant examples of new RE techniques and guidelines that have potential to change the way software is designed.

Acknowledgements We thank the authors for submitting high-quality manuscripts and thank the reviewers for this special issue for their time and constructive feedback, which helped to shape the articles. We also thank the Requirements Engineering journal and the Editor-in-Chief, Peri Loucopoulos, for their support of this special issue.

\section{Reference}

1. Damian DE, Perini A, Lee S-W (2019) 27th IEEE International Requirements Engineering Conference, RE 2019, Jeju Island, Korea (South), 23-27 Sept 2019. IEEE. ISBN 978-1-7281-3912-8.

Publisher's Note Springer Nature remains neutral with regard to jurisdictional claims in published maps and institutional affiliations. 